\title{
Initial Experience and Evaluation of Reusable Insulin Pen Devices Among Patients with Diabetes in Emerging Countries
}

Balduino Tschiedel $\cdot$ Oscar Almeida .

Jennifer Redfearn · Frank Flacke

To view enhanced content go to www.diabetestherapy-open.com

Received: July 30, 2014 / Published online: September 12, 2014

(C) The Author(s) 2014. This article is published with open access at Springerlink.com

\begin{abstract}
Background: Many individuals with type 2 diabetes in emerging countries are transitioning from vial-and-syringe insulin delivery to that of insulin pens (disposable or reusable). As with all insulin delivery methods, patient preferences and comfort are of utmost importance to optimize adherence to treatment. Patient-preferred characteristics for reusable insulin pens and barriers to appropriate injection, particularly in these regions, have not been widely reported in the clinical literature, highlighting a key information gap for clinicians considering these methods as part of

Electronic supplementary material The online version of this article (doi:10.1007/s13300-014-0081-z) contains supplementary material, which is available to authorized users.
\end{abstract}

B. Tschiedel $(\square)$

Institute for Children with Diabetes, R. Alvares Cabral, 529, Porto Alegre, RS 91350-250, Brazil

e-mail: badutsch@gmail.com

O. Almeida

Sanofi, Paris, France

J. Redfearn

The Research Partnership, Ltd., London, UK

F. Flacke

Sanofi, Frankfurt, Germany a comprehensive diabetes management approach.

Methods: Face-to-face interviews were conducted with people with type $1 / 2$ diabetes, including insulin-naïve and established insulin users. After moderator demonstration, participants were evaluated on their ability to perform a six-step process to inject a 10-unit dose into a pad with the AllStar ${ }^{\circledR}$ (AS; Sanofi, Mumbai, India), HumaPen Ergo II ${ }^{\circledR}$ (HE2; Eli Lilly, Indianapolis, USA), and NovoPen $4{ }^{\circledR}$ (NP4; Novo Nordisk, Bagsværd, Denmark) pens. Local pens were also tested in India, China and Brazil. Results: A total of 503 people from India, Malaysia, Brazil, Egypt, and China participated. Participants completed the sixstep process in an average, 2-3 min per pen. Participants ranked ease of overall use and ease of self-injection and dialing/reading dose as most important features for new insulin pens. When using the pens, the most difficult step was priming/safety testing, with $7-12 \%$ failing and $28-40 \%$ having difficulty; 6\%, 18\%, and $22 \%$ failed to hold the injection button down for the required period of time using AS, NP4, and HE2, respectively. Participants ranked AS significantly higher for nine of 12 ease-of-use 
features including three of the top four features considered the most important for reusable pens, while HE2 was ranked higher for two features. Local pens were ranked lowest.

Conclusions: Priming the pen and injecting the dose imparted most difficulty for people with diabetes in emerging countries. Most participants found AS easiest to use overall, with differences noted between pens for individual steps of dose delivery. Identifying characteristics most preferred by patients may assist in improving adherence to insulin therapy.

Keywords: AllStar; Diabetes; HumaPen; Insulin; Insulin pens; NovoPen

\section{INTRODUCTION}

The prevalence of diabetes is high in emerging countries or regions such as Brazil, India, China, the Middle East, and Southeast Asia and is expected to increase at least twofold in the next 20 years [1]. Most people with diabetes have type 2 diabetes mellitus (T2DM) and are initially treated with lifestyle changes and oral antidiabetic drugs [2]. However, because of the progressive nature of the disorder, many will need insulin to achieve glycemic control and thus, the use of insulin-based regimens is expected to continue to increase.

Some of the barriers to insulin use stem from the perceived discomfort and fear of insulin injections; these have been addressed by the introduction of insulin pens as a delivery mechanism [3]. Insulin pen design has focused on a combination of patient convenience/ satisfaction and affordability. This is especially important in emerging countries where the need for affordable, convenient insulin delivery is readily apparent. As patient awareness of insulin pens increases in emerging countries, it is important to identify those insulin pen characteristics that are most vital to optimizing patient acceptance and adherence. The aim of this market research survey is provide insight from people with diabetes in emerging countries into the usage characteristics of three to four different reusable insulin pens available in their respective countries, and to observe their pen usagebased preferences.

\section{METHODS}

\section{Patients}

Individuals with type 1 diabetes mellitus (T1DM) or T2DM were recruited for a market research survey in Brazil, China, Egypt, India, and Malaysia by various methods, including street intercept, snowball sampling (i.e., identifying one or more participants in the desired population and using them to find further participants), and recommendation by a healthcare professional. A mixture of gender, occupations, and insulin-naïve or insulinexperienced individuals were recruited. If patients were insulin naïve, they must have discussed starting insulin with their physician and must be willing to do so in the future, otherwise they were excluded from the study. People were also excluded if they had participated in diabetes market research in the past 3 months.

\section{Patient Interviews}

Face-to-face interviews were conducted in the local language in either the participant's home or a central location. Interviews were conducted from June to August 2013 by experienced 
moderators fully briefed on the research survey's objectives and materials (pens, cartridges, needles, instruction manuals), and the interview was recorded. After the interviewer confirmed information about the participant, each individual was given a shuffled deck of 12 cards, each showing a feature that could relate to a device for injecting a medicine for diabetes. The 12 key features were confirmed in a pilot phase consisting of 2 days of centrally viewed interviews in each market conducted earlier in May 2013. The participants were asked to put the 12 cards in order of importance to them. Each rank was assigned its corresponding numerical value (i.e., 1.0 for most important, 2.0 for second most important, and so on). For the entire group, the rankings were then averaged to determine the most important characteristics, with importance ranked from lowest to highest value.

The moderator then demonstrated the use of one of the devices, and the participants were asked to prepare the device and inject a 10-unit dose into a pad. This was repeated for each available device, and a manual in the local language was provided for each pen for reference. For each pen, participants were evaluated on their ability to (1) remove the cap, unscrew the cartridge holder and insert the cartridge; (2) screw/lock the cartridge holder; (3) attach the needle; (4) prime/safety test the device; (5) dial the dose; and (6) deliver the dose. The AllStar ${ }^{\circledR}$ (AS; Sanofi, Mumbai, India), NovoPen $4^{\circledR}$ (NP4; Novo Nordisk, Bagsværd, Denmark), and HumaPen Ergo II $^{\circledR}$ (HE2; Eli Lilly, Indianapolis, USA) were tested in all markets; in addition, one local pen was tested in India (INSUPen ${ }^{\circledR}$; Biocon, Bangalore, India), Brazil (HumaPen ${ }^{\circledR}$ Luxura $^{\mathrm{TM}}$; Eli Lilly, Sao Paulo, Brazil), and China (Xuilin Pen ${ }^{\circledR}$; Gan \&
Lee, Beijing, China). The order of presentation of the devices was randomized via three-way or four-way randomization (based on number of pens tested) so that a particular pen was not always first and two pens did not always follow one another. In all countries, participants were not tested on a device that they had used or were currently using, except in Malaysia. NP4 was tested by a sub-sample of current users in Malaysia $(n=52)$, where the pen is provided free of charge by the government.

The participants were next asked to compare each device across 12 key features by answering a series of questions (Table 1). They were asked to choose which pen was the best and worst for each of those features. Finally, they were asked which device was easiest to use overall, and which device was their last choice. For each question, participants in Brazil, China, and India were also asked to name their second choice, after naming their first choice, before naming their last choice. The results were tabulated and data were tested at the 95\% significance level, using $z$ tests and $t$ tests (as appropriate) to establish significance between means and proportions, respectively. Data were analyzed using QPSMR CL (version 2011.2, NetMR Ltd., Cupar, Fife, Scotland, UK) and DigitabXL (version 12.0, Digitab, London, UK).

As a market research survey, analyses herein do not fall under the auspices of the ethical standards on human experimentation (institutional and national) and the Helsinki Declaration of 1975 (revised 2000, 2008). Consent was gained from all patients for their participation in the demonstration of injection devices (following instructions from the moderators and presentation of instruction manuals) for the data to be reported at an aggregate level; patients were also informed about adverse events reporting procedures. 
Table 1 Questionnaire: comparison of devices

1. Which device is easiest for you to inject yourself? Which is hardest?

2. Which device is easiest for you to dial the right dose? Which is hardest?

3. Which device is easiest for you to read the dose? Which is hardest?

4. Which device do you think is the best if you needed to inject a high dose (e.g., 80 units)? Which is worst?

5. Which device is easiest for you to see how much insulin is in the cartridge? Which is hardest?

6. Which device is easiest in terms of feeling the dial turn and hearing the audible clicks telling you that it is working properly? Which is hardest?

7. Which device is the best size for you to hold in your hand when performing an injection? Which is worst?

8. Which device is the best weight for you to hold in your hand when performing an injection? Which is worst?

9. Which device is the most discreet and easy to carry? Which is worst?

10. Which device is easiest for you to change the cartridge? Which is hardest?

11. Which device is easiest for you to dial back (i.e., if you accidentally dial too much and need to reduce the dose, before injecting)? Which is hardest?

12. Which would you choose if you were thinking specifically about how the pen feels to hold, when you inject (e.g., the weight, the size, the ease of the injection button)? Which would be your last choice?

13. Thinking about all of the different features of these devices that we have discussed today, which device do you think is easiest to use, overall? Which would be your last choice?

\section{RESULTS}

\section{Patients}

A total of 503 participants from India, Malaysia, Brazil, Egypt, and China were interviewed (Table 2). The participants were equally divided between those who were either insulin naïve (49\%) or insulin users (51\%). More of the insulin users had T2DM (58\%) than had T1DM $(42 \%)$ due to only a few of the insulin users in Malaysia having T1DM (13\%). In the other four countries, insulin users were equally divided between those with T1DM and those with T2DM.

\section{Ease of Use of Pens}

Participants completed the preparation and dose injection process with AS, NP4, or HE2 in an average of 2-3 min (Fig. 1), with no significant differences between insulin-naïve participants and insulin users. There were differences between the pens for individual steps. For removing the cap, unscrewing the cartridge holder, and inserting the cartridge (Step 1), 20\% of participants struggled to remove the cartridge holder from NP4 due to its stiffness or to trying to pull the cartridge holder out rather than unscrew it; with HE2, 5\% of participants tried to twist the cap off and $2 \%$ had difficulty inserting the cartridge; with AS, $2 \%$ had difficulty inserting the cartridge into the pen/holder (e.g., inserting cartridge upside down). Three percent of participants using AS and 6\% using NP4 struggled to position the cartridge holder correctly (Step 2), while 5\% with HE2 had some difficulty with this step because of the stiff cartridge holder. Help to attach the needle (Step 3) was needed for $2 \%$ of 
Table 2 Distribution of participants by country and type of diabetes

\begin{tabular}{|c|c|c|c|c|c|c|}
\hline & Brazil & China & Egypt & India & Malaysia & Total \\
\hline Participants recruited, $n$ & 100 & 100 & 100 & 100 & 103 & 503 \\
\hline Type 1 diabetes & 23 & 25 & 25 & 25 & 8 & 106 \\
\hline Type 2 diabetes (insulin naïve) & 50 & 51 & 50 & 50 & 48 & 249 \\
\hline Type 2 diabetes (insulin users) & 27 & 24 & 25 & 25 & 47 & 148 \\
\hline \multicolumn{7}{|l|}{ Current pen (\% users) } \\
\hline NovoPen 2 or 3 & & & & & & 29 \\
\hline NovoPen 4 & & & & & & 38 \\
\hline HumaPen Ergo II & & & & & & 16 \\
\hline HumaPen other ${ }^{a}$ & & & & & & 10 \\
\hline AllStar (India only) & & & & & & 8 \\
\hline Other & & & & & & 2 \\
\hline \multicolumn{7}{|l|}{ Injections/day (\% users) } \\
\hline 1 Injection & & & & & & 20 \\
\hline 2 Injections & & & & & & 55 \\
\hline 3 Injections & & & & & & 14 \\
\hline 4 Injections & & & & & & 11 \\
\hline \multicolumn{7}{|l|}{ Age distribution, $n$} \\
\hline $18-30$ & 6 & 21 & 28 & 9 & 10 & 84 \\
\hline $31-40$ & 24 & 26 & 23 & 49 & 14 & 136 \\
\hline $41-50$ & 23 & 33 & 25 & 25 & 27 & 133 \\
\hline $51-60$ & 26 & 16 & 19 & 11 & 42 & 114 \\
\hline $61-65$ & 7 & 4 & 5 & 4 & 10 & 30 \\
\hline $65+$ & 4 & - & - & 2 & - & 6 \\
\hline \multicolumn{7}{|l|}{ Participants testing each pen, $n$} \\
\hline AllStar & 99 & 100 & 100 & 80 & 103 & \\
\hline HumaPen Ergo II & 89 & 92 & 97 & 71 & 103 & \\
\hline HumaPen Luxura & 93 & - & - & - & - & \\
\hline NovoPen 4 & 94 & 89 & 83 & 75 & 86 & \\
\hline Xuilin Pen & - & 99 & - & - & - & \\
\hline INSUPen & - & - & - & 78 & - & \\
\hline
\end{tabular}

a Ergo, Savio or Luxura 

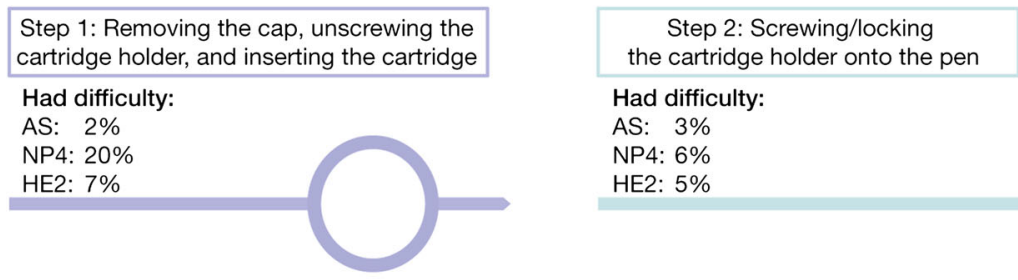

Step 3: Attaching the needle

Had difficulty:

AS: $2 \%$

NP4: $4 \%$

HE2: $6 \%$

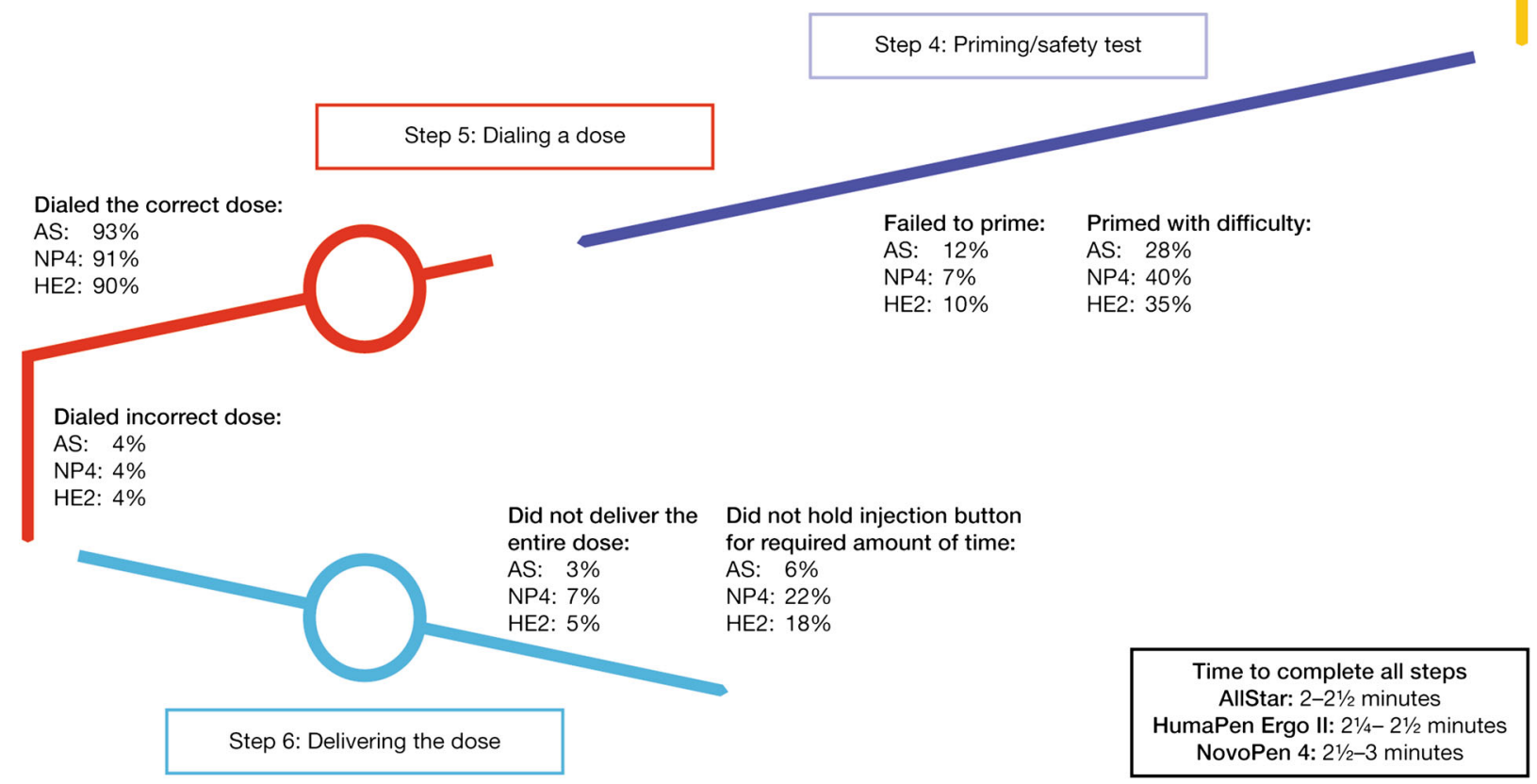

Fig. 1 Ease of use of AllStar, NovoPen 4, and HumaPen Ergo II pens-by steps. AS AllStar, HE2 HumaPen Ergo II, NP4 NovoPen 4

participants with AS, $4 \%$ with NP4, and $6 \%$ with HE2. In addition, $3 \%$ of insulin-naïve participants struggled with the needle safety cap with AS and 5\% struggled with NP4, both due to unfamiliarity with the cap.

There were $7-12 \%$ of participants who failed to prime the devices (Step 4); participants skipping the priming/safety test ranged from $3 \%$ (China) to $11 \%$ (Egypt) for AS, $4 \%$ (Brazil) to $10 \%$ (Egypt) for NP4, and 2\% (China) to 16\% (Egypt) for HE2. A total of 424 participants (88\%) primed AS, while 397 (93\%) primed NP4, and 407 (90\%) primed HE2. Of those who did prime the devices, $28 \%, 40 \%$, and $35 \%$ had difficulty priming with AS, NP4, and HE2, respectively. More than $90 \%$ of all problems were due to misunderstanding of the methodology required to perform a step, and were not due to mechanical issues with the pen. The correct dose (Step 5) was dialed by $90-93 \%$ of participants; $4 \%$ of participants dialed an incorrect dose with each pen, while the remainder either was unable to dial a dose (either due to dose button jam or forgetting to twist/pull out the dose button) or asked which dose to dial. Most participants delivered the entire dose (Step 6) with AS (97\%), NP4 (93\%) or HE2 (95\%), with significantly fewer participants failing to hold down the button for the required time with AS (6\%), compared with NP4 (22\%) 
and HE2 (18\%). Most participants knew an entire dose was delivered because the dose window read zero (all pens) and/or the dose button could not be depressed further (AS, NP4, and HE2) or the clicking sound stopped (NP4 and HE2).

\section{Most Important Features of an Insulin Pen}

Participants ranked ease of injecting oneself, ease of dialing the correct dose, how easy the device was to use overall, and ease of reading the dose display as the most important ease-ofuse features of any new insulin pen (Table 3 ). Being discreet and easy to carry was ranked as the least important of the 12 ease-of-use features. There were no significant differences between insulin-naïve and current insulin users in the top four characteristics.

Participants ranked AS highest on nine of 12 key features (Fig. 2), including three of the four most important features: easiest to use overall $(52 \%)$, easiest to read the dose $(42 \%)$, and easiest to self-inject (39\%). HE2 was ranked highest for easiest to dial back the dose (47\%) and to feel/hear dial clicks (39\%). It was also ranked highest for easiest to dial the right dose (36\%) but not significantly higher than AS (35\%). Local pens available in Brazil, China, and India were ranked the lowest for all 12 features. When asked which pen they would choose, if they were thinking specifically about how the pen feels to hold when they inject, $54 \%$ chose AS compared with 15\% for NP4, 23\% for HE2, and $8 \%$ for the local pen $(P<0.05$ for AS vs. all other pens).

\section{DISCUSSION}

The observations from this market research survey revealed, in a non-clinical setting, how people with diabetes in the emerging countries

Table 3 Most to least important features of a new insulin pen: by participant preference

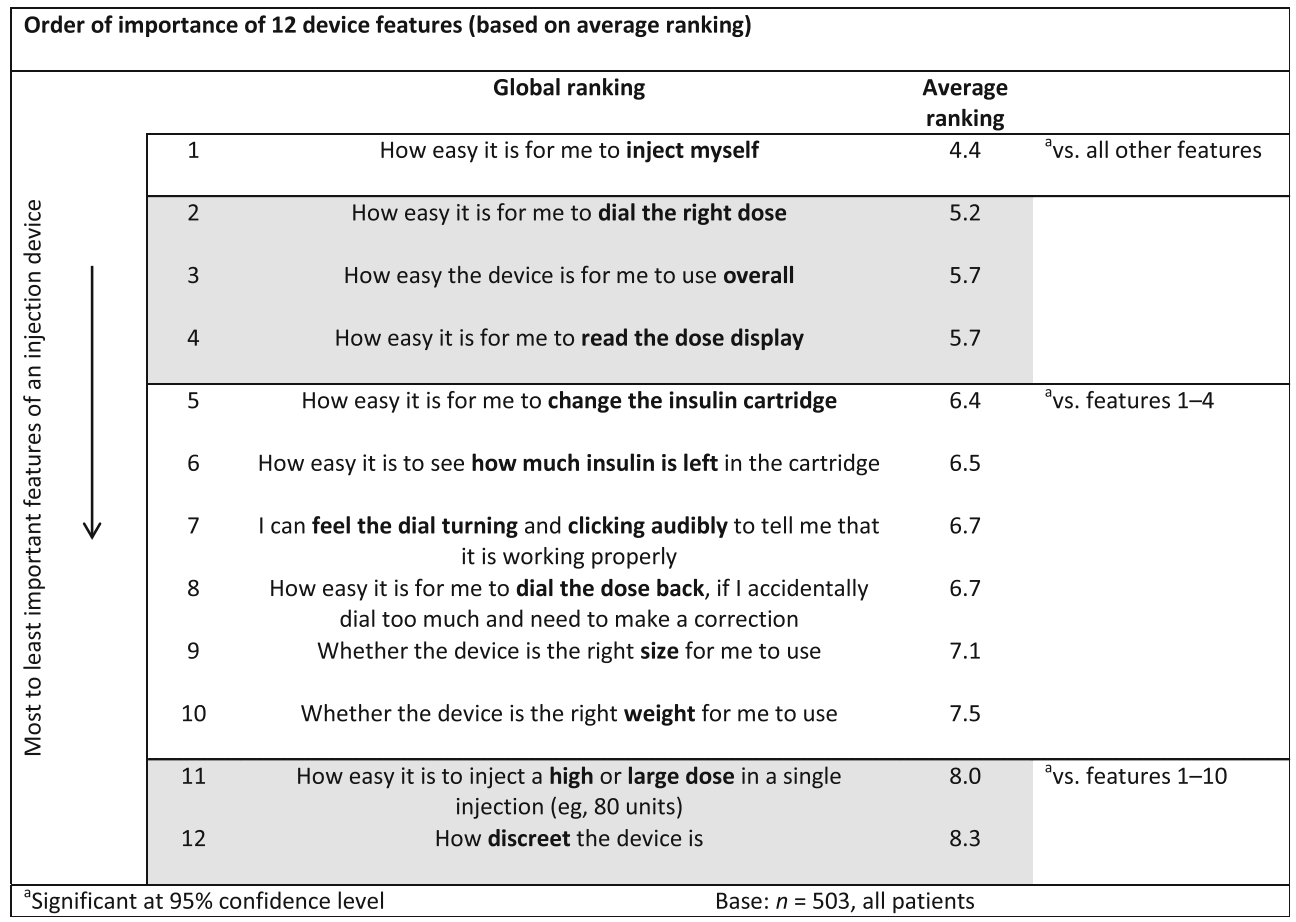




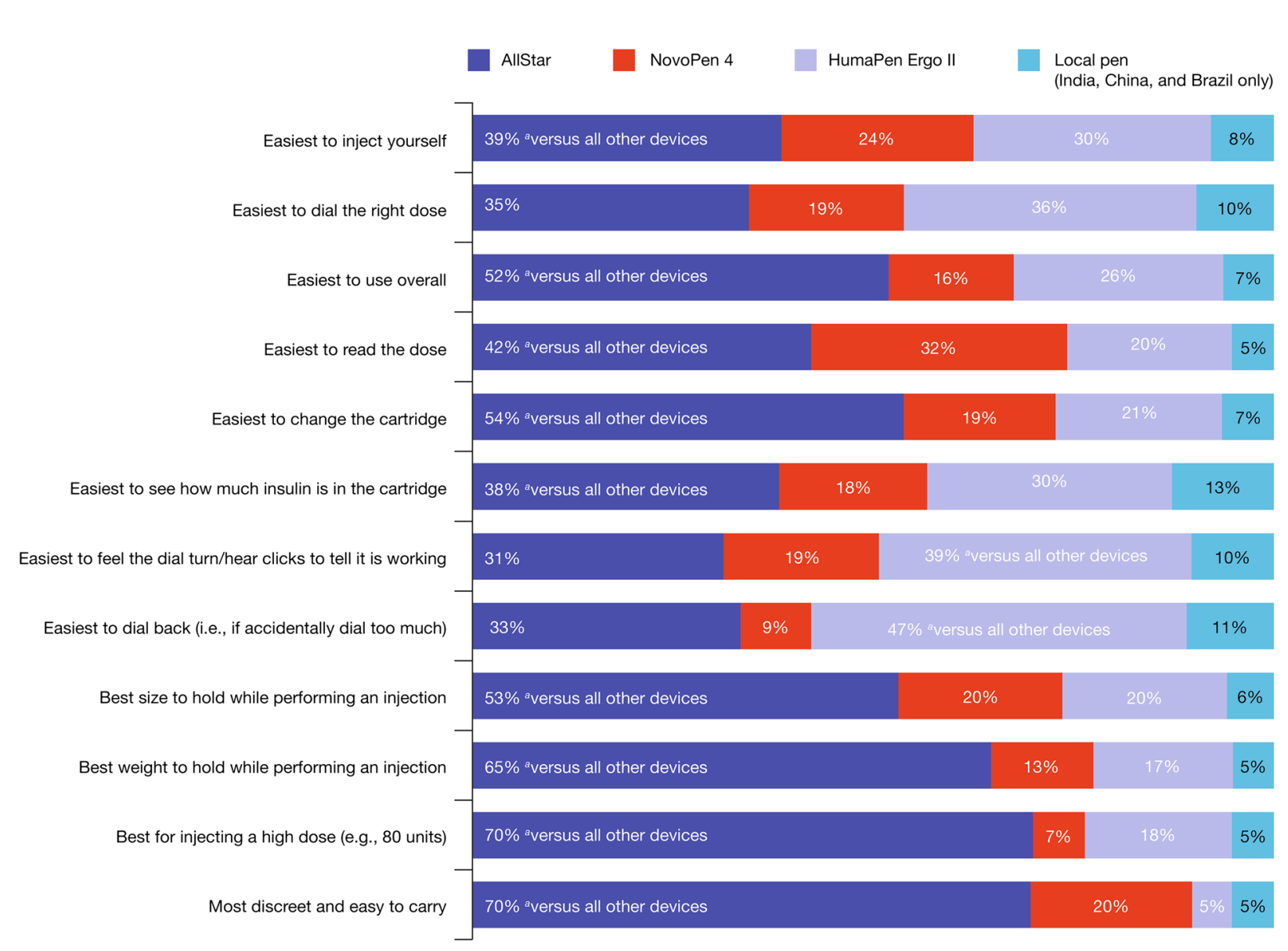

Fig. 2 Percentage of participants ranking each device in first place (globally)

of Brazil, China, Egypt, India, and Malaysia used the reusable insulin pens available in their country. Three pens-AS, NP4, and HE2-were available in all five countries, while an additional local pen was available in India, Brazil, and China. Most participants found the AS, NP4, and HE2 pens easy to use overall, but with some differences between pens in the individual steps used to inject a dose. The AS pen was ranked highest for nine of 12 ease-ofuse features, while HE2 was ranked highest for two features.

In the first part of the interviews, participants performed the sequential steps used to inject a dose of insulin once per pen. For insulin-naïve participants, this task had the possibility of being the easiest for the last pen tried as they learned from the preceding pens. To reduce this bias, a Latin-squares design was used to balance the order in which the pens were introduced. The tasks were followed by a question-and-answer session to determine the pen preferences of each participant. This design has been used in clinical studies involving insulin pens [4-6] and allows for a comparison of pen features without any influence from the insulin therapy. This may mimic clinical practice for many people with diabetes, where a diabetes educator or nurse would offer the person the opportunity to test and choose from a selection of devices appropriate for the prescribed insulin regimen. Clinical studies have evaluated pen devices over an extended period of actual use with insulin [7-11], and 
evaluation of pen features in many of these studies may have been influenced by the therapeutic outcome with the pen.

Most people with diabetes who require insulin self-administer it by subcutaneous injection, using either a vial and syringe or an insulin pen device. Worldwide, pen devices are used by approximately $60 \%$ of insulin users, although usage varies from country to country [12]. Among the countries involved in the current study, $95 \%$ of people with diabetes in China are pen users, whereas approximately $60 \%$ and $80 \%$ use vial and syringe in Brazil and India, respectively. Compared with vial and syringe, insulin pens provide better dosing accuracy, easier dosing and administration, convenience, increased patient acceptance and satisfaction; they are also discreet and easily portable, which lessens social embarrassment [13-17]. Pens are considered less painful than syringes and are associated with less needle fear [17]. Their use is also linked with improved adherence to insulin therapy and reduced costs compared with vial and syringe [18], and may reduce the resistance to starting insulin therapy.

The results provided herein give important clinical insights into the preferences and usage capabilities of individuals in regions of the world where reusable insulin pen availability is extensive and/or increasing. From a clinical perspective, the ability of an individual to feel confident and comfortable with the use of such a device is an essential factor in enhancing patient adherence to diabetes management. As insulin injections are a daily requirement, recognizing the attributes most desirable to the patient and providing a device that best fits those preferences help to ensure seamless integration of insulin delivery to a patient's everyday routine. Additionally, remaining cognizant of those steps that potentially pose difficulty with each pen is also important, so that the diabetes management team (whether physician, nurse, or caregiver) can focus their instruction on those steps which may be most problematic. This holds true for both newly initiated insulin users (where pen characteristics may factor in treatment choice) as well as experienced insulin pen users (for their current insulin treatment, or if a change in treatment is considered).

Potential limitations to this study include first-impression preferences by participants without long-term, learned pen use, as well as the inability to gauge comfort and execution of actual self-injection, particularly for those who have never utilized insulin. As such, areas of further research and discussion if data were available include: (1) influence of age: younger versus older participants; (2) insulin-naïve participants versus insulin users; and (3) T1DM vs. T2DM participants.

\section{CONCLUSIONS}

As a result of interviews with individuals with T1DM or T2DM, it was identified that new and existing users of insulin pens seek ease of injection, overall ease of use, and correct dose delivery as key characteristics for an insulin pen device. Through hands-on use of these different pens, priming the reusable insulin pens was the most difficult aspect of administering a dose; however, each pen showed slight variation in the steps that posed difficulty with administration. The AS pen was easiest to use overall compared with other reusable pens tested, and ranked highest by uses in most of the characteristics identified as most preferred for a reusable insulin pen. Selection of an appropriate reusable insulin pen may provide benefit and comfort for patients starting or continuing insulin therapy; identifying those 
characteristics that are most preferred by patients may assist in overcoming barriers to appropriate dose delivery and overall adherence with treatment.

\section{ACKNOWLEDGMENTS}

This study was conducted by The Research Partnership, Ltd. Sponsorship and article processing charges for this study were funded by Sanofi, Bridgewater, USA. Editorial support was provided by Tom Claus, PhD, of PPSI, Hackensack, USA (a PAREXEL company) and was funded by Sanofi. All named authors meet the ICMJE criteria for authorship for this manuscript, take responsibility for the integrity of the work as a whole, and have given final approval for the version to be published.

Conflict of interest. Balduino Tschiedel is a consultant to Sanofi, Roche Diagnostics, Novo Nordisk and Eli Lilly; Jennifer Redfearn is a consultant to Sanofi; Oscar Almeida is an employee of Sanofi; Frank Flacke is an employee of Sanofi.

Compliance with ethics guidelines. As a market research survey, analyses herein do not fall under the auspices of the ethical standards on human experimentation (institutional and national) and the Helsinki Declaration of 1975 (revised 2000, 2008). Consent was gained from all patients for their participation, and they were informed about adverse events reporting procedures.

Open Access. This article is distributed under the terms of the Creative Commons Attribution Noncommercial License which permits any noncommercial use, distribution, and reproduction in any medium, provided the original author(s) and the source are credited.

\section{REFERENCES}

1. International Diabetes Federation. IDF Diabetes Atlas, 6th ed. Belgium: International Diabetes Federation. http://www.idf.org/diabetesatlas. Accessed March 24, 2014.

2. Inzucchi SE, Bergenstal RM, Buse JB, Diamant M, Ferrannini E, Nauck M, Peters AL, Tsapas A, Wender $\mathrm{R}$, Matthews DR, American Diabetes Association (ADA), European Association for the Study of Diabetes (EASD). Management of hyperglycemia in type 2 diabetes: a patient-centered approach: position statement of the American Diabetes Association (ADA) and the European Association for the Study of Diabetes (EASD). Diabetes Care. 2012;35:1364-79.

3. Magwire ML. Addressing barriers to insulin therapy: the role of insulin pens. Am J Ther. 2011;18:392-402.

4. Asakura T, Seino H. Assessment of dose selection attributes with audible notification in insulin pen devices. Diabetes Technol Ther. 2005;7:620-6.

5. Fox C, McKinnon C, Wall A, Lawton SA. Ability to handle, and patient preference for, insulin delivery devices in visually impaired patients with type 2 diabetes. Pract Diabetes Int. 2002;19:104-7.

6. Haak T, Edelman S, Walter C, Lecointre B, Spollett G. Comparison of usability and patient preference for the new disposable insulin device Solostar versus Flexpen, lilly disposable pen, and a prototype pen: an open-label study. Clin Ther. 2007;29:650-60.

7. D'Eliseo P, Blaauw J, Milicevic Z, Wyatt J, Ignaut DA, Malone JK. Patient acceptability of a new $3.0 \mathrm{ml}$ pre-filled insulin pen. Curr Med Res Opin. 2000;16:125-33.

8. Graff MR, McClanahan MA. Assessment by patients with diabetes mellitus of two insulin pen delivery systems versus a vial and syringe. Clin Ther. 1998;20:486-96.

9. Korytkowski M, Bell D, Jacobsen C, Suwannasari R. A multicenter, randomized, open-label, comparative, two-period crossover trial of preference, efficacy, and safety profiles of a prefilled, disposable pen and conventional vial/ syringe for insulin injection in patients with type 1 or 2 diabetes mellitus. Clin Ther. 2003;25:2836-48.

10. Ristic S, Bates PC, Martin JM, Llewelyn JA. Acceptability of a reusable insulin pen, HumaPen Ergo, by patients with type 1 and type 2 diabetes. Curr Med Res Opin. 2002;18:68-71. 
11. Stocks A. HumaPen Ergo: a new $3.0 \mathrm{~mL}$ reusable insulin pen. Clin Drug Invest. 2001;21:319-24.

12. Perfetti R. Reusable and disposable insulin pens for the treatment of diabetes: understanding the global differences in user preference and an evaluation of inpatient insulin pen use. Diabetes Technol Ther. 2010;12(Suppl 1):S79-85.

13. Dang DK, Lee J. Analysis of symposium articles on insulin pen devices and alternative insulin delivery methods. J Diabetes Sci Technol. 2010;4:558-61.

14. Goldstein HH. Pen devices to improve patient adherence with insulin therapy in type 2 diabetes. Postgrad Med. 2008;120:172-9.

15. Luijf YM, Devries JH. Dosing accuracy of insulin pens versus conventional syringes and vials. Diabetes Technol Ther. 2010;12(Suppl 1):S73-7.
16. Pfutzner A, Asakura T, Sommavilla B, Lee W. Insulin delivery with FlexPen: dose accuracy, patient preference and adherence. Expert Opin Drug Deliv. 2008;5:915-25.

17. Anderson BJ, Redondo MJ. What can we learn from patient-reported outcomes of insulin pen devices? J Diabetes Sci Technol. 2011;5:1563-71.

18. Asche CV, Shane-McWhorter L, Raparla S. Health economics and compliance of vials/syringes versus pen devices: a review of the evidence. Diabetes Technol Ther. 2010;12(Suppl 1):S101-8. 